\section{Case Reports in Ophthalmology}

\title{
Tuberculous Subretinal Abscess in a Non-HIV Patient with Miliary Tuberculosis
}

\author{
Eugenia Paez Soria ${ }^{a} \quad$ Paula Magnano $^{a} \quad$ Ariel Schlaen $^{a} \quad$ Paula Luvini $^{b}$ \\ Gisela Arevalo Calderon ${ }^{c}$ Mauricio Martinez Cartier ${ }^{d}$ Pablo Chiaradía ${ }^{a}$ \\ Cristóbal Couto ${ }^{a}$ \\ ${ }^{a}$ Department of Ophthalmology, University of Buenos Aires, Buenos Aires, Argentina; \\ ${ }^{b}$ Department of Internal Medicine, University of Buenos Aires, Buenos Aires, Argentina; \\ 'Department of Infectious Diseases, University of Buenos Aires, Buenos Aires, Argentina; \\ ${ }^{\mathrm{d}}$ Instituto de la Vision, Buenos Aires, Argentina
}

\section{Keywords}

Subretinal abscess · Optical coherence tomography $\cdot$ Fundus autofluorescence $\cdot$ Miliary tuberculosis

\section{Abstract}

This case report describes the features of a tuberculous subretinal abscess in a non-HIV patient. It includes the characterization of the lesion with spectral-domain optical coherence tomography (SD-OCT) and fundus autofluorescence examination. SD-OCT and autofluorescence imaging showed external retinal involvement during development of the tuberculous subretinal abscess. Patients with tuberculous lesions need a multidisciplinary approach, since the disease may involve any part of the body. In this case in particular, incidental findings at computed tomography scan led to the detection of this rare ocular manifestation of tuberculous disease. 


\section{Introduction}

Tuberculosis (TB) is a granulomatous disease caused by Mycobacterium tuberculosis, which currently remains a worldwide health problem, especially in endemic countries and in patients undergoing any kind of immunosuppression [1]. In 2014, 9.6 million people have fallen ill and 1.5 million people died of TB [2].

Ninety percent of infected people are asymptomatic, while among those who develop the disease, close to $80 \%$ have pulmonary manifestations. Only $20 \%$ of the patients have an extrapulmonary disease. Ocular TB is a rare disorder. In cases with ophthalmic involvement, any part of the eye or the orbit can be affected. Posterior uveitis is the most common ocular presentation, particularly multiple choroidal tubercles [1].

We report a case of a young male with disseminated miliary TB who was referred to us because of the incidental finding of a tuberculous subretinal abscess in his left eye when a brain computed tomography (CT) was performed.

\section{Case Report}

A 24-year-old man was admitted to our hospital with major complaints of weight loss, asthenia, and a precervical mass during the last month. He had a family history of 2 of his relatives, who lived with him in an extremely poor environment, undergoing anti-TB medical treatment. A fine-needle aspiration biopsy of the mass was performed, and the Ziehl-Neelsen stain was positive for acid-fast bacilli. He was diagnosed as having scrofuloderma in the context of lung miliary TB. Bacilloscopy was negative on 3 occasions during hospitalization. Immunological status was assessed without remarkable results, and HIV test was negative. The patient, who had a nonbacilliferous TB, was started on treatment with isoniazid, rifampin, pyrazinamide, and ethambutol and, therefore, he was discharged after 1 week of hospitalization to continue ambulatory treatment.

Notwithstanding, he came back 3 weeks later with acute paraparesis. Clinical examination revealed prevertebral cervical and lumbar abscesses, and an urgent laminectomy was performed. His tuberculin skin test ( 5 tuberculin units $/ 0.1 \mathrm{~mL}$ ) was highly positive $(20 \times 25$ $\mathrm{mm}$ ), and his HIV screening remained negative. A severe malnutrition with a body mass index of 17 together with an important decrease in hematocrit and plasma proteins were observed. During his hospitalization, he continued with the standard 4-drug regimen for TB treatment. A pan-computed tomography detected hepatic abscesses, pleural effusion, pulmonary miliary TB, a brain abscess in the frontal lobe (Fig. 1), and a mass in the left eye (Fig. 2). The latter finding led to a consultation at our service.

At ophthalmic examination, the patient had a best-corrected visual acuity of 20/20 in $\mathrm{OD}$ and counting fingers in OS. Anterior segment examination had no remarkable signs. Dilated fundoscopy showed a solitary yellowish elevated choroidal lesion involving the optic disc in OS. Next to the lesion, peripapillary and macular folds were observed. The longest diameter of the lesion was 6 DD (Fig. 3).

An spectral-domain optical coherence tomography (SD-OCT; Spectralis, Heidelberg) was performed of the lesion (Fig. 4), where a hyperreflective choroidal mass with a hyporeflective center was seen. The line of retinal pigment epithelium/Bruch's membrane complex was disrupted, with a hyperreflective substance located in the subretinal space. A subretinal fluid over the hyperreflective substance could be seen, with membranous structures dividing the subretinal space into several compartments. The macula was uninvolved, although nasal 


\section{Case Reports in Ophthalmology}

retinal folds and a posterior hyaloid detachment nasally to the fovea were observed. Fundus autofluorescence revealed a hypoautofluorescent area corresponding to the tuberculoma, surrounded by a hyperautofluorescent perilesional halo (Fig. 5).

Based on the clinical examination and the images of the patient, the most likely presumptive diagnosis was a tuberculous subretinal abscess. Therefore, the decision was to continue with the 4-drug regimen for TB to complete a 3-month period and then with 2 drugs for an additional 9 months.

After 6 months of TB treatment, the abscess had decreased in size to form a flat and pigmented scar (Fig. 6). However, the visual acuity of his left eye remained without change, and he developed a remarkable exotropia. During a 2-year period of follow-up, the lesion remained inactive without further changes.

\section{Discussion}

Tuberculous subretinal abscess is an infrequent presentation of ocular tuberculosis. It develops from liquefaction necrosis from caseous material of large tuberculomas [3]. Tuberculomas can be seen in patients with miliary TB [4-7], tuberculous central nervous system involvement [8-10], TB of the spine [4], and immunosuppressed patients [11,12]. It has also been reported in association with AIDS, and a minimal inflammatory reaction may be observed when it occurs [13]. Tuberculous subretinal abscesses have been described in HIV/AIDS [14] but also in immunocompetent patients [15]. In the case described in this report, involvement of multiple organs occurred, including pulmonary miliary tuberculosis and paravertebral, brain, and liver abscesses.

Abscesses are more yellow than choroidal tuberculomas and often have overlying retinal hemorrhages. In patients with disseminated tuberculosis, these lesions can be associated with slight overlying vitreal inflammation $[1,3]$.

There is 1 description of OCT for a tuberculous subretinal abscess. In a case report of a peripapillary subretinal abscess associated with posterior scleritis, OCT showed gross disk edema and cystoid macular edema [15]. However, there is no description of the external retinal and choroidal damage as in our case. According to the features observed on the OCT images of our patient, we can infer that the former choroidal lesion disrupted Bruch's membrane and the pigment retinal epithelium, invading the subretinal space. Subretinal membranous structures likely correspond to fibrin formation, dividing the subretinal space into several compartments.

To our knowledge, currently, there are no reports on fundus autofluorescence imaging for tuberculous subretinal abscesses. The hypoautofluorescence observed at the surface of the subretinal abscess may be caused by the inflammatory destruction of the retinal pigment epithelium and the presence of a subretinal inflammatory exudate.

As a conclusion, these patients need a multidisciplinary approach, since the disease may involve any part of the body. In this case in particular, incidental findings on CT scan led to the detection of this rare ocular manifestation of the disease. OCT and autofluorescence imaging showed the external retinal involvement during the development of the tuberculous subretinal abscess. 
Paez Soria et al.: Tuberculous Subretinal Abscess in a Non-HIV Patient with Miliary Tuberculosis

\section{Funding Sources}

No financial support was received for this submission.

\section{Statement of Ethics}

The study was performed with informed consent and following all the guidelines for experimental investigations required by the institutional review boards or ethics committees with which all authors are affiliated.

\section{Disclosure Statement}

None of the authors has any conflict of interest in connection with this submission.

\section{References}

1 Gupta V, Gupta A, Rao NA: Intraocular tuberculosis - an update. Surv Ophthalmol 2007;52:561-587.

2 Organization WH: Global Tuberculosis Report 2015. Geneva, WHO Press, 2015.

-3 Shetty SB, Bawtag MA, Biswas J: A case of subretinal tubercular abscess presenting as disc edema. Indian J Ophthalmol 2015;63:164-166.

4 Annamalai R, Biswas J: Bilateral choroidal tuberculoma in miliary tuberculosis - report of a case. I Ophthalmic Inflamm Infect 2015;5:4.

5 Yilmaz T, Selcuk E, Polat N, Mutlu K: Choroidal tuberculoma showing paradoxical worsening in a patient with miliary TB. Ocul Immunol Inflamm 2015;23:97-99.

6 Sharma PM, Singh RP, Kumar A, Prakash G, Mathur MB, Malik P: Choroidal tuberculoma in miliary tuberculosis. Retina 2003;23:101-104.

7 Padhi TR, Basu S, Das T, Samal B: Optic disc tuberculoma in a patient with miliary tuberculosis. Ocul Immunol Inflamm 2011;19:67-68.

-8 Nor-Masniwati S, Zunaina E, Azhany Y: Ocular tuberculosis with multiple cerebral abscesses. Case Rep Ophthalmol Med 2012;2012:606741.

-9 Zhang M, Zhang J, Liu Y: Clinical presentations and therapeutic effect of presumed choroidal tuberculosis. Retina 2012;32:805-813.

10 Laktaoui A, Naoumi A, Reda K, Kriet M, Elouarsani A, Khayati A, Elharradi A, Rouimi A, Douhal A Mounahc J, Houmadi A: Macular tuberculoma and optic neuritis: rare association with tuberculosis meningoencephalitis (in French). J Fr Ophtalmol 2009;32:673-678.

11 Han BG, Choi SO, Lee SJ, Kim YH, Hong WP, Lee JH: Choroidal tuberculoma with membranous glomerulonephritis. Yonsei Med J 2001;42:446-450.

$\checkmark 12$ Atmaca L, Yalcindag FN, Ciledag A: Choroidal tuberculoma in a patient with ocular Behcet disease. Int Ophthalmol 2012;32:93-96.

$\checkmark 13$ DiLoreto DA Jr, Rao NA: Solitary nonreactive choroidal tuberculoma in a patient with acquired immune deficiency syndrome. Am J Ophthalmol 2001;131:138-140.

14 Babu RB, Sudharshan S, Kumarasamy N, Therese L, Biswas J: Ocular tuberculosis in acquired immunodeficiency syndrome. Am J Ophthalmol 2006;142:413-418.

15 Pappuru RR, Dave VP: An unusual case of ocular tuberculosis presenting as subretinal abscess with posterior scleritis. Int Ophthalmol 2016, DOI: 10.1007/s10792-016-0254-z. 


\section{Case Reports in Ophthalmology}

\begin{tabular}{l|l}
\hline Case Rep Ophthalmol 2016;7:292-300 \\
\hline DOI: 10.1159/000453447 & $\begin{array}{l}\text { C } 2016 \text { The Author(s). Published by S. Karger AG, Basel } \\
\text { www.karger.com/cop }\end{array}$ \\
\hline
\end{tabular}

Paez Soria et al.: Tuberculous Subretinal Abscess in a Non-HIV Patient with Miliary Tuberculosis

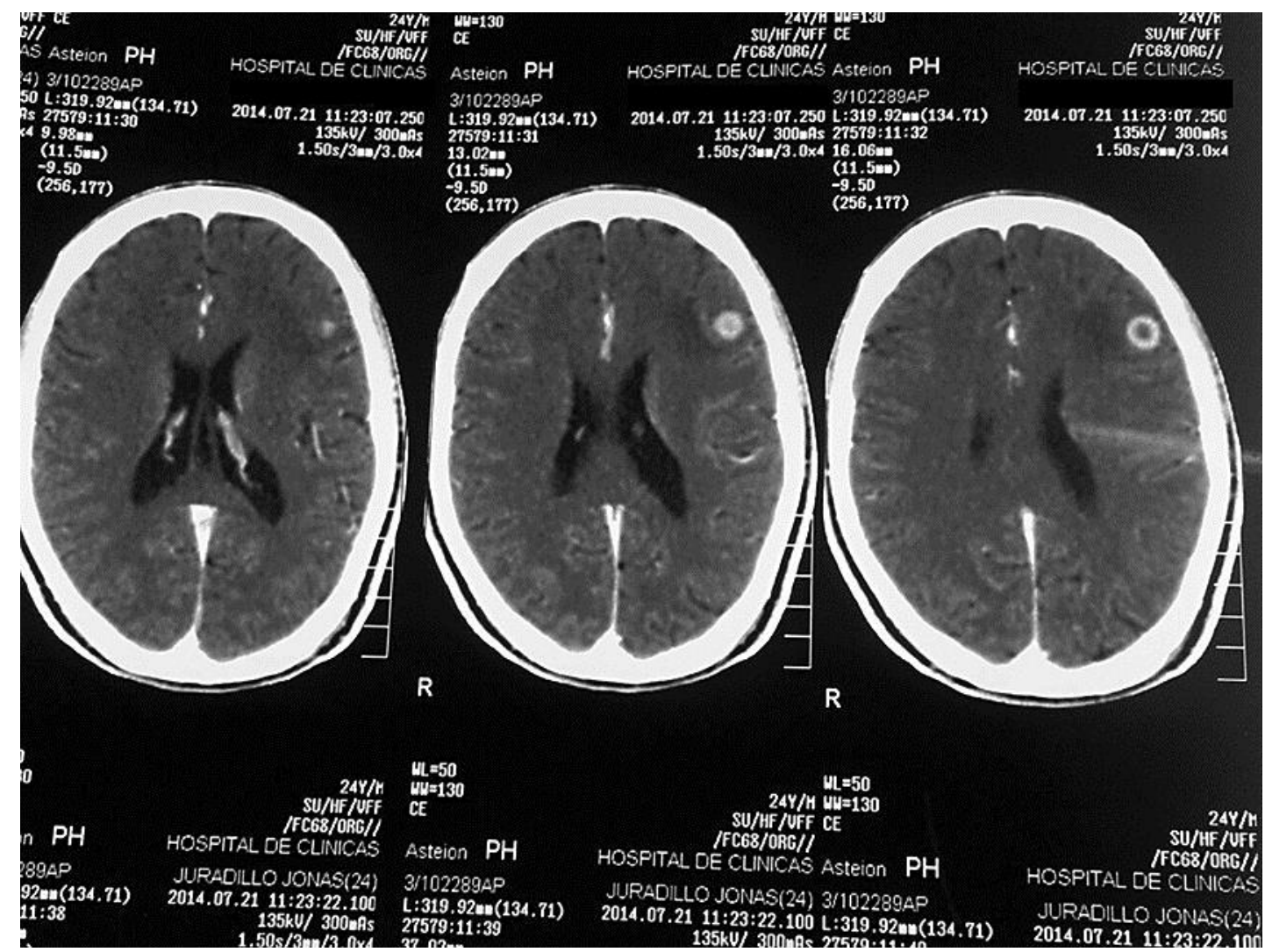

Fig. 1. Computed tomography of the brain: note the brain abscess in the left frontal lobe. 


\section{Case Reports in Ophthalmology}

\begin{tabular}{l|l}
\hline Case Rep Ophthalmol 2016;7:292-300 \\
\hline DOI: 10.1159/000453447 & $\begin{array}{l}\text { @ 2016 The Author(s). Published by S. Karger AG, Basel } \\
\text { www.karger.com/cop }\end{array}$ \\
\hline
\end{tabular}

Paez Soria et al.: Tuberculous Subretinal Abscess in a Non-HIV Patient with Miliary Tuberculosis

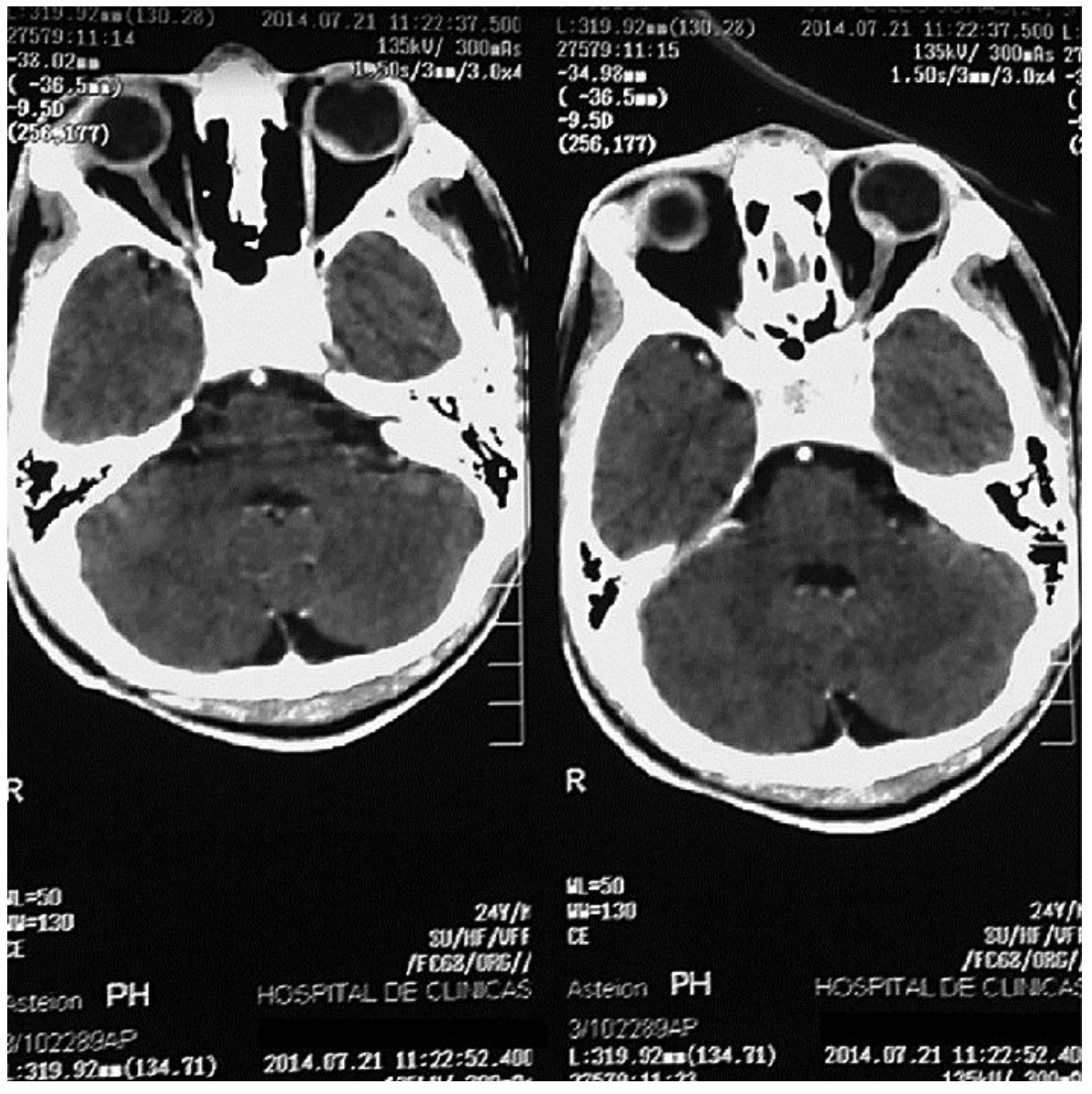

Fig. 2. Computed tomography of the orbit: note the mass on the posterior wall of the left eye. 


\section{Case Reports in Ophthalmology}

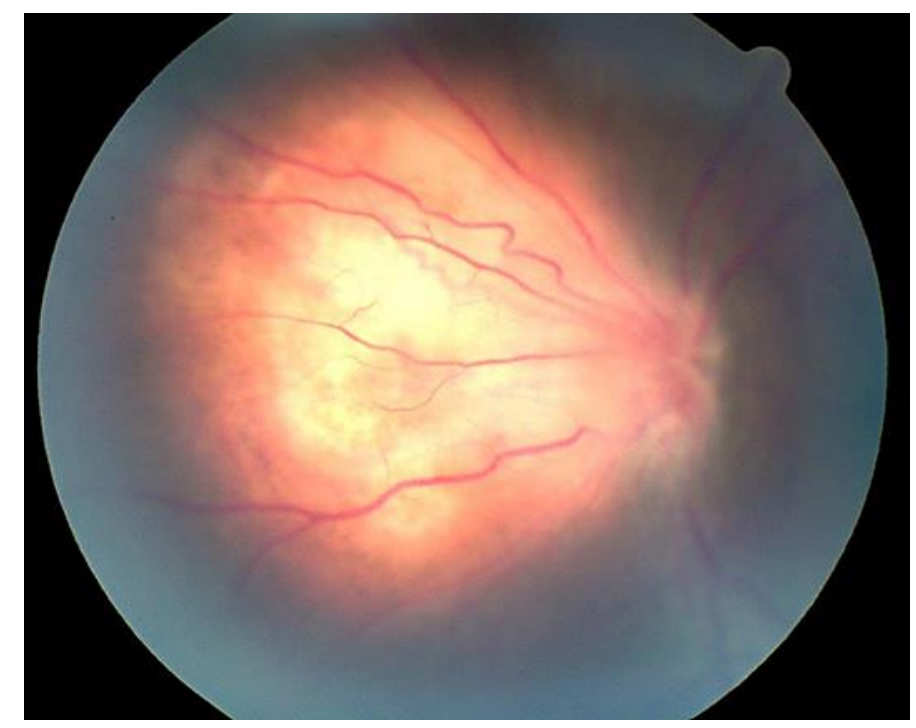

Fig. 3. Fundus photography showing choroidal tuberculoma in the posterior pole. A yellowish choroidal mass can be seen, extending nasally from the optic disk and involving it. Note the peripapillary folds associated with the lesion.

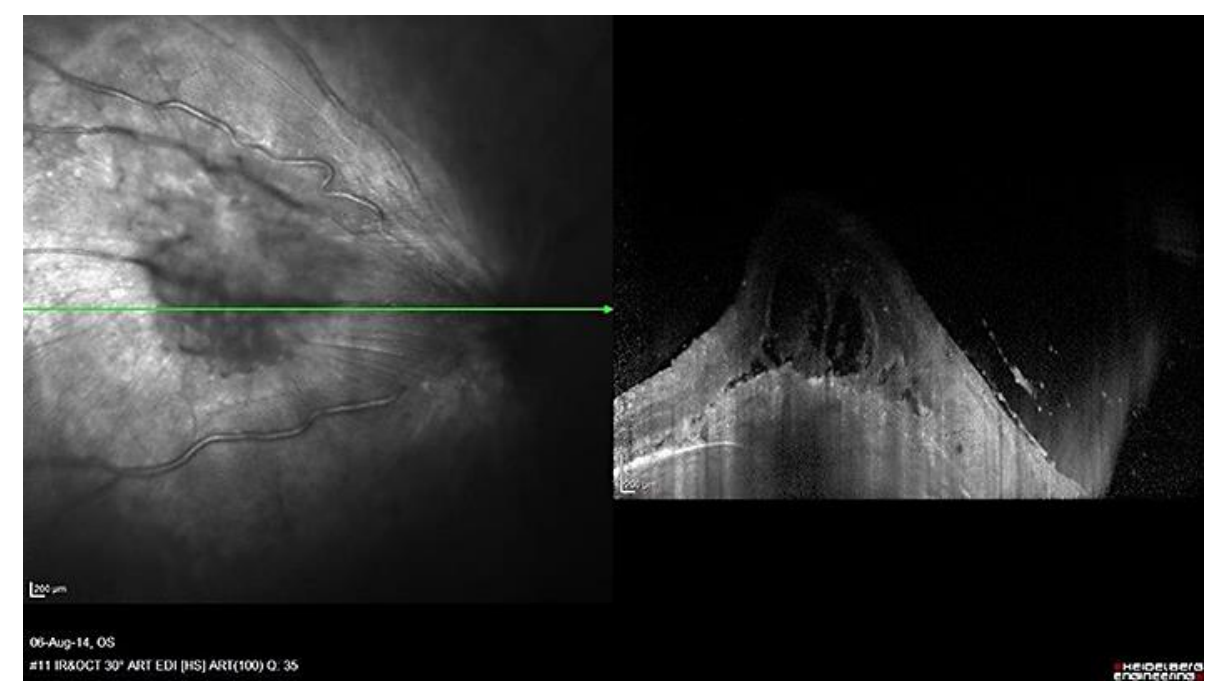

Fig. 4. Spectral-domain optical coherence tomography image showing a juxtapapillary subretinal abscess. The line of retinal pigment epithelium/Bruch's membrane complex was disrupted, with a hyperreflective substance located in the subretinal space. A subretinal fluid over the hyperreflective substance can be seen, with membranous structures dividing the subretinal space into several compartments. 
Case Reports in
Ophthalmology

Case Rep Ophthalmol 2016;7:292-300

(c) 2016 The Author(s). Published by S. Karger AG, Basel DOI: $10.1159 / 000453447$ www.karger.com/cop

Paez Soria et al.: Tuberculous Subretinal Abscess in a Non-HIV Patient with Miliary Tuberculosis

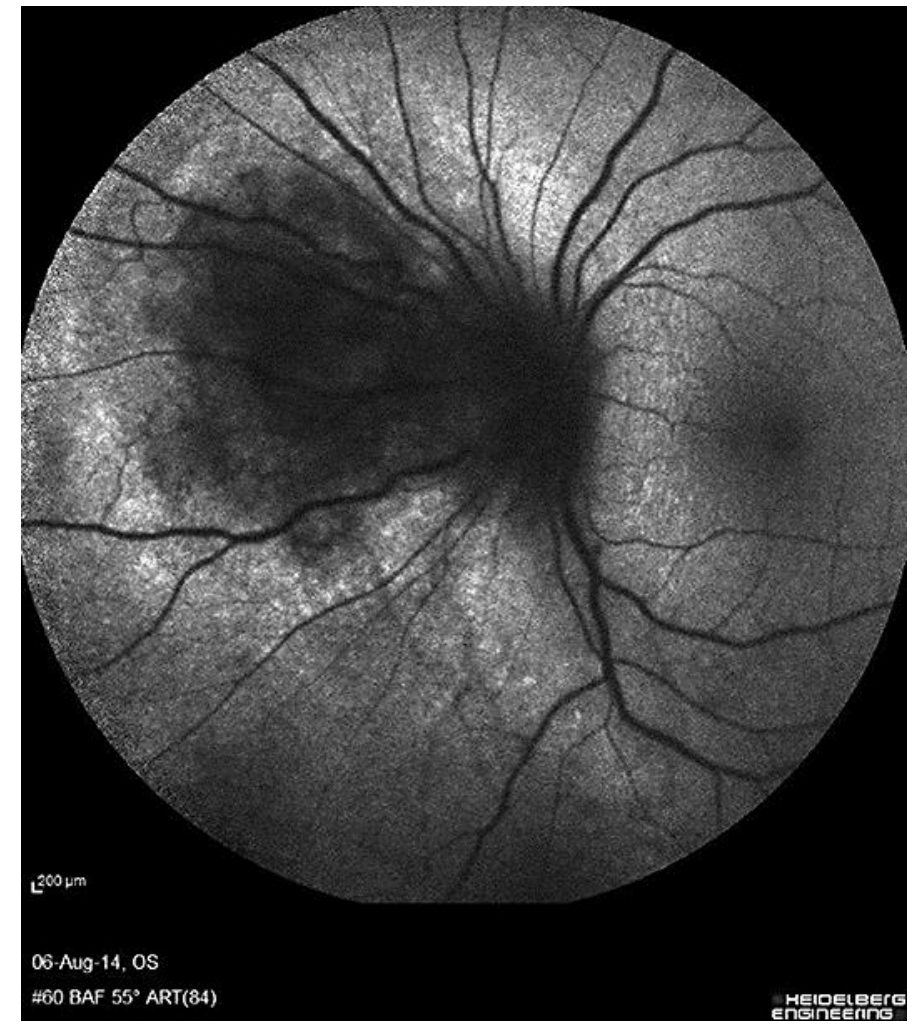

Fig. 5. Fundus autofluorescence showing the location and size of the lesion. A hypoautofluorescent area, corresponding to the tuberculoma, surrounded by a hyperautofluorescent perilesional halo can be observed. 
Case Reports in
Ophthalmology

Case Rep Ophthalmol 2016;7:292-300

(c) 2016 The Author(s). Published by S. Karger AG, Basel DOI: $10.1159 / 000453447$ www.karger.com/cop

Paez Soria et al.: Tuberculous Subretinal Abscess in a Non-HIV Patient with Miliary Tuberculosis

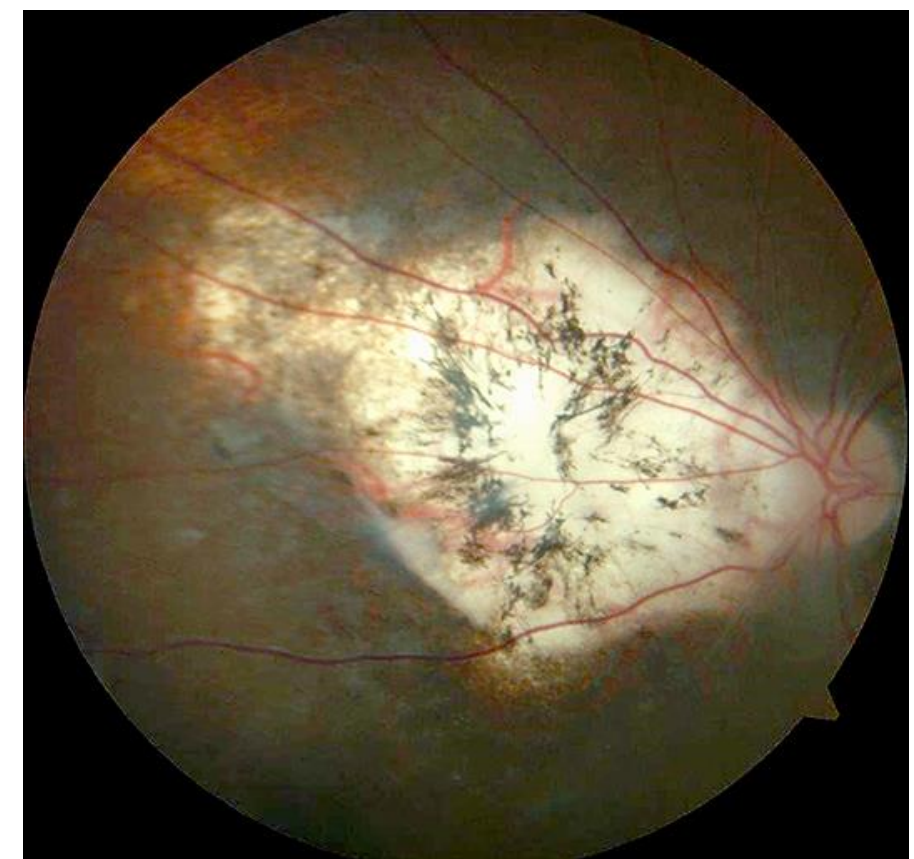

Fig. 6. Fundus photography showing an extensive pigmented scar nasally from the optic disc. 